\title{
Schrödinger's dentist
}

Shaun Sellars continues his essential series on ethical dilemmas in dentistry which appears in

every second issue of the $B D J$.

2021 arrived with a hush rather than

the usual bang. As I write, we're in our third national lockdown, which is due to continue until at least mid-February but will in all likelihood go on for much longer. Things are different for many of us as we pick up our probes and return to the enamel face. General dentists who have an NHS contract now have to produce over twice as much activity as they did towards the end of last year, all against a backdrop of rising coronavirus infections and a new, highly transmissible virus variant.

This leaves dentists facing a tough choice: increase their patient contact and turnover, or prepare for the inevitable clawback of contracted payments from the NHS. Understandably, there's a large kickback against this from many and a number of those working in private practice appear enraged over the 'scandal' of NHS dentists not pulling their weight. It would seem that the dental practice is either the safest place to be or too dangerous, depending on who pays your way.
Seeing, and being, a dentist at the moment is relatively safe - but not risk-free - and the various components that contribute to that risk are changing rapidly. Each patient and staff contact carries with it an element of risk. With a more transmissible virus, that risk becomes greater. Combine this with the increase in UDA targets for NHS practices, and the danger to dental staff and patients will undoubtedly rise.

So, in relation to before, dentistry will be riskier, but this increase in relative risk tells us very little about how (un)safe dentistry really is. Much as when the media reports that the contraceptive pill triples the risk of deep vein thrombosis, they handily miss out that the baseline risk is small and even tripling this gives a very low absolute risk.

Unfortunately, there are few data on the transmission of coronavirus in the dental setting, so unlike the contraceptive pill, we have no idea of the baseline risk, apart from it being low. Because of this uncertainty, it's hard to estimate the increase in absolute risk of the ramping up of UDA targets. We

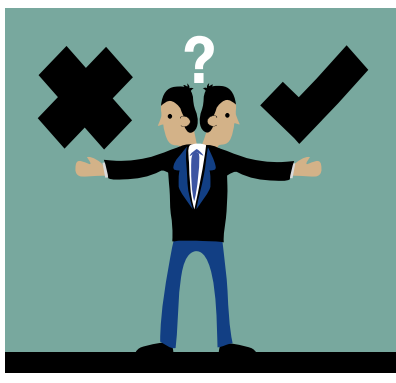

can be sure that both patients and staff members are more likely to pick up SARSCoV-2 from the outside world than in the dental surgery, leading to practices potentially being unable to provide full nursing or clinical cover to see patients, or appointment books being too empty because patients are showing symptoms or feel that their non-urgent appointment need not go ahead given the guidance to stay at home. Still, going to the dentist is safer than visiting the supermarket, less safe than staying on the sofa - but staying on the sofa doesn't spot that oral cancer that's developing.

Given these new challenges, the increase in UDA targets for practices appears imprudent. I'd expect this to be reconsidered, perhaps even before this is published, because it would appear that dentists can be incredibly safe, but too dangerous at the same time.

\section{DCT creates wellbeing whiteboards}

Sunmeet Kandhari, a DCT2 in restorative dentistry at Birmingham Dental Hospital, has written to the $B D J$ to share a wellbeing initiative he has started in his workplace to boost team morale during the COVID-19 pandemic.

Sunmeet writes: 'It's a simple premise. Essentially, I organised for a large freestanding whiteboard to be set up in the hospital entrance, which I add motivational and inspirational quotes to daily. So far, staff, student, and patient feedback has been overwhelmingly positive and there are now plans to roll out further boards trust-wide. I have also been contacted by a few dental students in King's for advice in setting up their own boards'.

Two examples of Sunmeet's 'thought for the day'-style boards are pictured here.
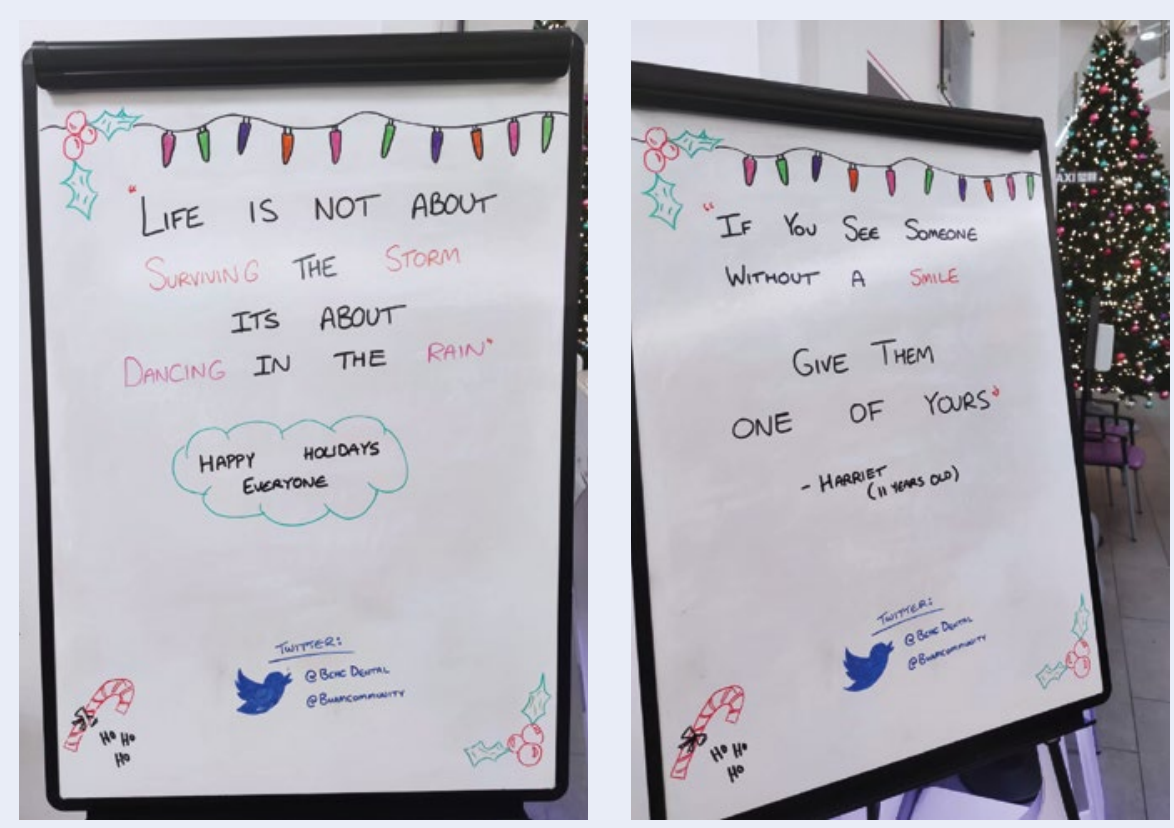